Original paper

\title{
Homogeneous vs. patient specific breast models for Monte Carlo evaluation of mean glandular dose in mammography
}

\author{
A. Sarno ${ }^{a}$, G. Mettivier ${ }^{a}, *, F$. Di Lillo ${ }^{a}$, K. Bliznakova ${ }^{b}$, I. Sechopoulos ${ }^{c}$, P. Russo ${ }^{a}$ \\ a Dipartimento di Fisica "Ettore Pancini", Università di Napoli Federico II \& INFN Sez. di Napoli, I-80126 Napoli, Italy \\ ${ }^{\mathrm{b}}$ Laboratory of Computer Simulations in Medicine, Technical University of Varna, Varna, Bulgaria \\ ${ }^{\mathrm{c}}$ Department of Radiology and Nuclear Medicine, Radboud University Medical Centre and Dutch Expert Centre for Screening (LRCB), Nijmegen, Netherlands
}

\section{A R T I C L E I N F O}

\section{Keywords:}

Breast models

Mean glandular dose

Mammography

Monte Carlo simulations

\begin{abstract}
A B S T R A C T
Purpose: To compare, via Monte Carlo simulations, homogeneous and non-homogenous breast models adopted for mean glandular dose (MGD) estimates in mammography vs. patient specific digital breast phantoms.

Methods: We developed a GEANT4 Monte Carlo code simulating four homogenous cylindrical breast models featured as follows: (1) semi-cylindrical section enveloped in a 5-mm adipose layer; (2) semi-elliptical section with a 4-mm thick skin; (3) semi-cylindrical section with a 1.45-mm skin layer; (4) semi-cylindrical section in a 1.45-mm skin layer and 2-mm subcutaneous adipose layer. Twenty patient specific digital breast phantoms produced from a dedicated CT scanner were assumed as reference in the comparison. We simulated two spectra produced from two anode/filter combinations. An additional digital breast phantom was produced via BreastSimulator software.

Results: With reference to the results for patient-specific breast phantoms and for W/Al spectra, models \#1 and \#3 showed higher MGD values by about $1 \%$ (ranges $[-33 \% ;+28 \%]$ and $[-31 \% ;+30 \%]$, respectively), while for model \#4 it was $2 \%$ lower (range [ $-34 \%$; $+26 \%]$ ) and for model \#2 -11\% (range $[-39 \%$; $+14 \%]$ ), on average. On the other hand, for W/Rh spectra, models \#1 and \#4 showed lower MGD values by 2\% and 1\%, while for model \#2 and \#3 it was $14 \%$ and $8 \%$ lower, respectively (ranges [ $-43 \%$; $+13 \%]$ and [ $-41 \%$; $+21 \%]$ ). The simulation with the digital breast phantom produced with BreastSimulator showed a MGD overestimation of $+33 \%$.

Conclusions: The homogeneous breast models led to maximum MGD underestimation and overestimation of 43\% and $28 \%$, respectively, when compared to patient specific breast phantoms derived from clinical CT scans.
\end{abstract}

\section{Introduction}

The dose reference parameter in 2D mammography is the mean glandular dose (MGD) [1], i.e. the dose, on average, to the glandular tissue of the breast undergoing the exam. Following several protocols worldwide [2-5], the MGD is estimated from measurement of the air kerma at the entrance skin surface of the compressed breast, multiplied by suitable conversion coefficients: the normalized glandular dose (DgN) coefficients [6,7] or the set of $(\mathrm{g}, \mathrm{c}, \mathrm{s})$ coefficients [8,9]. These coefficients depend on the beam characteristics, as well as on the breast anatomy, such as the compressed thickness and the glandular fraction. Both DgN and (g, c, s) coefficients are computed via Monte Carlo (MC) simulations where the breast is suitably modelled. In the current protocols (see the review in Ref. [5]), the breast is represented as a cylinder with a semi-circular or semi-ellipsoidal section, made of a homogeneous mixture of glandular and adipose tissue enveloped in a layer mimicking the skin. These models replicate neither the real breast shape nor the heterogeneous texture of the glandular tissue. However, the glandular tissue in the breast departs from the nipple and is mainly located in the central part of the organ, surrounded by the adipose tissue: this layer may partially shield the inner radiosensitive tissue. Dance et al. [10] showed that the location of the gland within the breast could bring to MGD differences as large as $48 \%$, pointing out the lack of 3D digital breast models with a realistic glandular tissue distribution for a proper dose calculation. Modern 3D breast imaging techniques - such as computed tomography dedicated to the breast (BCT) [11-14] - allowed producing patient-like digital breast models, which reflect the breast anatomical characteristics for a more accurate MGD calculation [15]. Both Sechopoulos et al. [16] and Hernandez et al. [17] studied the influence of the homogeneous assumption on the calculated MGD. They produced digital compressed breast phantoms from high-resolution 3D images of breasts acquired via BCT scanners. These digital phantoms

\footnotetext{
* Corresponding author.

E-mail address: mettivier@na.infn.it (G. Mettivier).
} 
presented a heterogeneous glandular distribution, which mimicked that of real breasts. In addition, they generated homogeneous breast phantoms by substituting the heterogeneous tissue with a homogeneous one with the same glandular fraction. Via MC simulations, they found out that the MGD calculated with the so-modelled homogenous digital breast phantoms was about $30 \%$ higher, on average, with respect to the corresponding heterogeneous digital breast phantoms. In addition, in Ref. [16] the ratio between the MGD evaluated with the homogeneous and the heterogeneous models was comprised in the range 2.17 and 0.84 , so indicating a maximum overestimation and underestimation of $117 \%$ and $16 \%$, respectively, for the studied cohort. In any case, the models adopted in protocols for MGD estimates in mammography do not reproduce any real breast and present skin thicknesses and compositions, which differ from the homogeneous digital phantoms used in Refs. $[16,17]$. Indeed, the skin thickness and its composition have a huge impact on the MGD estimates, and the thicker is the skin, the lower is the dose to the gland, for the photon energies usually used in mammography $[18,19]$. While the digital phantoms derived from BCT images presented a skin thickness of $1.5 \mathrm{~mm}$ [17], the model proposed in Ref. [8] for the (g, c, s) calculation had a 5-mm thick skin made of adipose tissue. On the other hand, in Ref. [6] the skin had a thickness of $4 \mathrm{~mm}$. These differences in skin thickness as well as the breast shapes and dimensions may partially compensate for the MGD overestimation caused by the homogeneous assumption. In this respect we also note that the skin thickness may not be constant on the surface of the breast [18].

This work aimed at comparing the homogeneous breast models usually adopted for MGD estimates, with patient specific digital breast phantoms, which reflect both the anatomy and glandular tissue distribution of real breasts. These patient specific digital phantoms were obtained from segmented 3D images of the uncompressed breast acquired via a commercial BCT scanner and digitally compressed via a suitable mechanical model. In addition, a digital breast phantom was developed via BreastSimulator software package [20], for a preliminary investigation of its adoption in breast dosimetry in addition to breast imaging investigations.

\section{Materials and Methods}

\subsection{MC code}

A MC code for MGD calculation in X-ray breast imaging was developed and presented in previous papers [19,21-24]. It is based on GEANT4 ver. 10.00 toolkit and adopts the standard physics list Option4. The default photon threshold cutoffs were used, and photoelectric, coherent and incoherent scatter photon interactions were simulated. Due to the low influence on the MGD calculation $[19,21]$, the electrons were not tracked, but supposed to release energy at the generated location. The composition of the simulated breast tissues was that proposed in Ref. [1]; for water and PMMA the compositions indicated in the NIST database were used.

\subsection{Homogeneous breast models}

We investigated four homogeneous breast models with the following characteristics: Model \#1, a cylinder with a semi-circular section with a radius of $8 \mathrm{~cm}$ and a $0.5-\mathrm{cm}$-thick adipose layer surrounding the radiosensitive breast tissue; Model \#2, a cylinder with a semi-elliptical section with minor semi-axis - connecting the nipple to the chest - of $8 \mathrm{~cm}$ and the major axis of $18 \mathrm{~cm}$ and with $0.4-\mathrm{cm}$ thick skin; Model \#3, a cylinder with a semi-circular cross section with a radius of $10 \mathrm{~cm}$ and with $0.145-\mathrm{cm}$ thick skin; Model \#4, a cylinder with a semicircular cross section with a radius of $10 \mathrm{~cm}$ and with $0.145-\mathrm{cm}$ thick skin and 2-mm subcutaneous adipose layer. The inner portions of the four models were made of a homogeneous mixture of glandular and adipose tissue. The model \#1 reflects the breast geometry proposed in
Table 1

Homogeneous breast models investigated within this work.

\begin{tabular}{lllll} 
Model & Breast section & $\begin{array}{l}\text { Skin } \\
\text { layer } \\
(\mathrm{mm})\end{array}$ & $\begin{array}{l}\text { Adipose } \\
\text { layer }(\mathrm{mm})\end{array}$ & References \\
\hline$\# 1$ & Semi-circular; Radius $=8.0 \mathrm{~cm}$ & 0.00 & 5.00 & {$[8]$} \\
$\# 2$ & $\begin{array}{l}\text { Semi-ellipsoidal; Minor semi-axis } \\
\text { (chest-to-nipple) }=8.0 \mathrm{~cm}, \text { Major }\end{array}$ & 4.00 & 0.00 & {$[6]$} \\
& axis $=18.0 \mathrm{~cm}$ & & & \\
$\# 3$ & Semi-circular; Radius $=10.0$ & 1.45 & 0.00 & {$[18,19]$} \\
$\# 4$ & Semi-circular; Radius $=10.0$ & 1.45 & 2.00 & {$[19,25]$} \\
\hline
\end{tabular}

Ref. [8] and the model \#2 the one in Ref. [6].

However, literature [25] and recent research studies conducted with BCT scanners $[18,26]$ showed that the skin thickness is lower than that proposed in Ref. [8] (5 mm) and in Ref. [6] (4 mm). The thickness of the skin layer was between $0.5 \mathrm{~mm}$ and $2.0 \mathrm{~mm}$, and the subcutaneous adipose layer [25] was not visible in 3D images of the breast. Huang et al. [18], with a relatively large cohort of imaged breasts, showed that the breast skin presents an average thickness of $1.45 \mathrm{~mm}$, much less than the thickness adopted in Refs. [6,8]. Shi et al. [26] reached similar results ( $1.44 \mathrm{~mm}$ of average thickness) with an independent patient cohort. For this reasons, in this study, we investigated also the models \#3 and \#4 described above. Table 1 summarizes the specifications of the homogeneous breast models investigated in this paper. The breast thickness and glandular fraction by mass were selected on the basis of the characteristics of the compared patient specific breast.

\subsection{BreastSimulator}

BreastSimulator is a modular software package for breast imaging research [20]. It is composed by the following four main modules: 1) Breast Modeling Module for generating 3D breast models; 2) Breast Compression Module for compressing digital breast models; 3) Image Generation Module for analytically generating synthetic projection images; 4) Visualization Module composed of a set of utilities to visualize 2D and 3D breast images. The module 1 permits to create digital breast phantoms with a selected composition. The simulated breasts' features include the breast shape, the duct system, the Cooper's ligaments, the pectoralis muscle, the skin and the lymphatic and blood systems; in addition, breast lesions can be added [27]. The user can increase the complexity of the breast model by including any of such features and by increasing their number or size. Mettivier et al. [27] showed a first validation of this software vs. real 3D breast images in terms of anatomical noise in a previous work.

BreastSimulator's module 2 permits to simulate the mechanical compression (based on the algorithm described in Ref. [28]) of the 3D uncompressed breast models. The 3D breast images (whose voxels are classified in adipose tissue, glandular tissue and skin tissue) are divided into model elements each consisting of 27 voxels. They are connected via springs, having uniform and isotropic linear modulus of elasticity. The software permits to classify the voxels on the basis of their content [29]. The tissue was considered as incompressible; hence, the breast volume does not change during the compression. The Young's modulus for the skin, the adipose tissue and the glandular tissue were set to $88 \mathrm{kPa}, 1 \mathrm{kPa}$ and $10 \mathrm{kPa}$, respectively [30].

\subsection{Digital breast phantom via BreastSimulator}

A digital breast phantom was generated via the BreastSimulator software, for MGD calculation and comparison to patient specific digital breast phantom (Fig. 1). Its glandular fraction by mass was $16 \%$ and it was compressed, via the module 2 of BreastSimulator, in order to have a compressed breast thickness of $68.6 \mathrm{~mm}$. The dimension of the voxels was $0.245 \times 0.245 \times 0.245 \mathrm{~mm}^{3}$. Differently from the homogeneous 


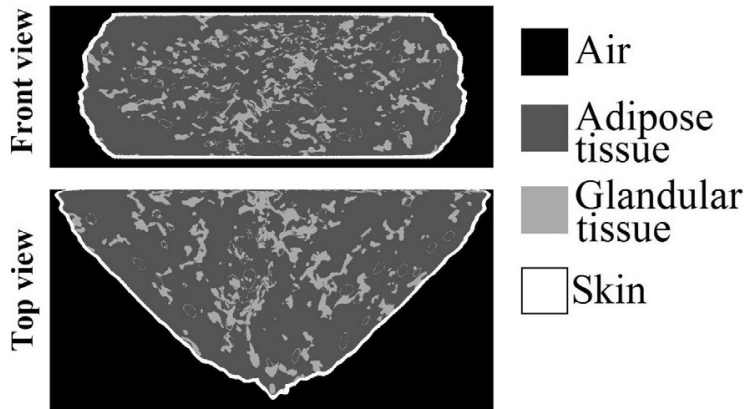

Fig. 1. Compressed digital breast phantom produced via BreastSimulator.

breast models presented in the previous sections, the digital phantom produced with BreastSimulator has the glandular tissue heterogeneously distributed within the adipose tissue. The phantom voxels content was classified as skin tissue, adipose tissue or gland, by using the algorithm described in Ref. [29]. The skin thickness at the upper surface of the breast ranged between $1.470 \mathrm{~mm}$ and $1.715 \mathrm{~mm}$ after the compression process and the breast footprint was 208,578 pixels $\left(12,519.89 \mathrm{~mm}^{2}\right)$.

The used phantom was picked-up from a pre-computed digital phantom dataset. It replicates at best the characteristics of one of the patient specific breast phantoms involved in this paper. The use of this single digital phantom represents only a preliminary study to test the use of the digital breast phantoms produced via BreastSimulator package for breast dosimetry. We plan to extend the study for a larger cohort of digital phantoms, for mimicking the large variation in 3D glandular dose distribution.

\subsection{Patient specific breast phantoms}

In order to validate the homogeneous models presented in the Section 2.3, we used 20 patient specific 3D digital phantoms. They have already been presented in Ref. [16] and adopted in Ref. [22]. They reproduced the anatomy and shape characteristics of the breast undergoing compression during mammographic examinations (Fig. 2). The inner part of the breast was a heterogeneous mixture of glandular tissue and adipose tissue, mimicking the spatial distribution within a real breast. The 20 digital phantoms were produced from 3D images of 20 pendulant breasts acquired with the Koning Corp. BCT scanner (http://koninghealth.com/en/). In this case, the voxels were classified in 4 categories on the basis of their content: air, glandular tissue, adipose tissue and skin tissue [31]. Consequently, the uncompressed breast phantoms were compressed via the mechanical model adopted in the BreastSimulator package described in Section 2.3. The compressed shape and anatomy reflected those of the breasts undergoing mammographic examinations in CC view [28]. The thicknesses of the compressed breasts were selected on the basis of the thickness presented by the same patients' breasts when submitted to mammographic exams. Table 2 summarizes the characteristics of the 20 digital phantoms after the compression.

\subsection{MGD calculation}

The MGD in the case of homogeneous model was computed as $[19,21,22]$ :

$M G D=\frac{\sum_{i} G_{i}(E) \times E_{i}^{d e p}}{f_{g} \times W_{b}}$

where $E_{\mathrm{i}}^{\mathrm{dep}}$ is the energy absorbed at the interaction event $i, f_{\mathrm{g}}$ is the breast glandular fraction by mass, and $W_{\mathrm{b}}$ is the breast mass (skin excluded). The factor $G(E)$, introduced in Ref. [7] introduces the probability to have energy absorbed in the glandular tissue within a homogeneous mixture:

$G(E)=\frac{f_{\mathrm{g}} \times \frac{\mu_{\mathrm{en}}}{\rho}(E)_{\mathrm{g}}}{f_{\mathrm{g}} \times \frac{\mu_{\mathrm{en}}}{\rho}(E)_{\mathrm{g}}+\left(1-f_{\mathrm{g}}\right) \times \frac{\mu_{\mathrm{en}}}{\rho}(E)_{\mathrm{a}}}$

Here, $\mu_{\mathrm{en}} / \rho$ is the mass energy absorption coefficient of glandular (subscript $g$ ) and adipose (subscript $a$ ) tissues, evaluated by considering the functional interpolation given in Ref. [32]. The $G$-factor was evaluated interaction-by-interaction, at the current energy of the photon during the transport process [33].

On the other hand, in the cases where heterogeneous breast phantoms were adopted, we scored the sole energy absorbed in the glandular tissue. Then we divided it by the total glandular mass comprised in the breast, in order to calculate the MGD.

\subsection{Irradiation geometry and technique factors}

The simulated X-ray source is an isotropic point source placed at $64.5 \mathrm{~cm}$ from the upper part of the breast support paddle. The beam was collimated via software in order to irradiate a surface of $24 \times 30 \mathrm{~cm}^{2}$ at $1.5 \mathrm{~cm}$ below the breast lower surface. The breast support paddle and the top breast compression paddle were $24 \times 30 \mathrm{~cm}^{2}$ plates of PMMA with a thickness of $0.2 \mathrm{~cm}$. In all simulations, a $30 \times 30 \times 15 \mathrm{~cm}^{3}$ box of water was included in order to simulate the patient body. The irradiation geometry is represented in Fig. 3.

The X-ray beam was simulated as a polyenergetic beam mimicking the one emitted from a $\mathrm{W}$ anode either with a $0.700 \mathrm{~mm}$ of added filtration of $\mathrm{Al}(\mathrm{W} / \mathrm{Al})$ or $0.050 \mathrm{~mm}$ of added filtration of rhodium (W/

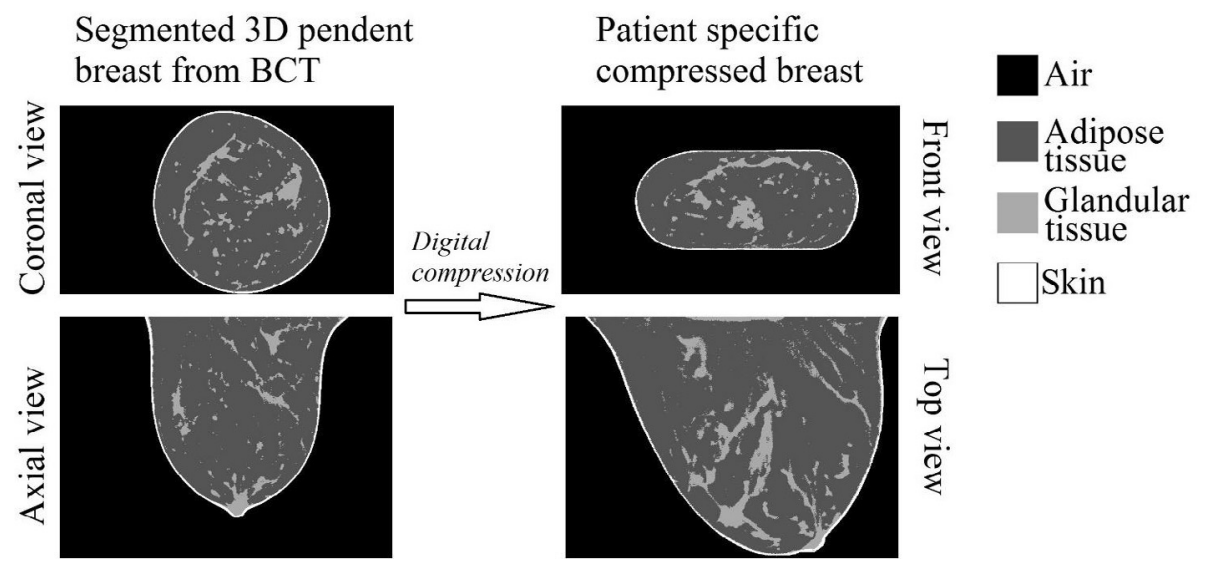

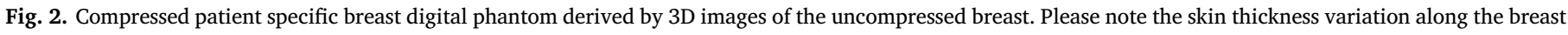
silhouette. 
Table 2

Characteristics of the 20 patient specific digital breast phantoms.

\begin{tabular}{|c|c|c|c|c|c|c|c|}
\hline & Mean & Std. Dev. & Min & Max & Median & 1st quartile & 3rd quartile \\
\hline Glandular fraction by mass (\%) & 23.1 & 15.3 & 5.0 & 54.3 & 17.6 & 10.7 & 32.4 \\
\hline Compressed breast thickness $(\mathrm{cm})$ & 5.9 & 1.5 & 2.9 & 7.8 & 6.1 & 5.0 & 7.2 \\
\hline
\end{tabular}

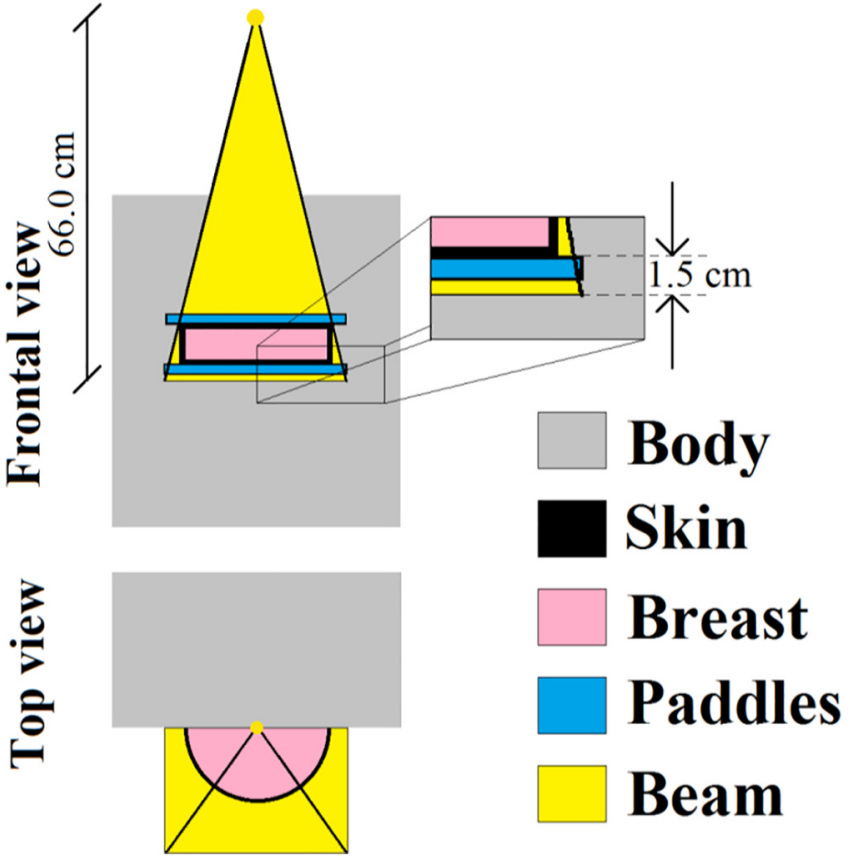

Fig. 3. Irradiation geometry adopted in this work.

Table 3

Technique factors used in this work for breast irradiations.

\begin{tabular}{|c|c|c|c|c|c|}
\hline \multicolumn{6}{|c|}{ Technique factors } \\
\hline \multirow{2}{*}{$\begin{array}{l}\text { Breast } \\
\text { thickness } \\
\text { range } \\
(\mathrm{cm})\end{array}$} & \multirow{2}{*}{$\begin{array}{l}\text { Tube } \\
\text { voltage } \\
(\mathrm{kV})\end{array}$} & \multicolumn{2}{|c|}{$\mathrm{W} / 0.700 \mathrm{~mm} \mathrm{Al}$} & \multicolumn{2}{|c|}{$\mathrm{W} / 0.050 \mathrm{~mm} \mathrm{Rh}$} \\
\hline & & $\begin{array}{l}\text { Simulated } \\
\text { HVL (mm } \\
\mathrm{Al})\end{array}$ & $\begin{array}{l}\text { Calculated } \\
\text { HVL below } \\
\text { the } \\
\text { compression } \\
\text { paddle (mm } \\
\text { Al) }\end{array}$ & $\begin{array}{l}\text { Simulated } \\
\text { HVL (mm } \\
\text { Al) }\end{array}$ & $\begin{array}{l}\text { Calculated } \\
\text { HVL below } \\
\text { the } \\
\text { compression } \\
\text { paddle (mm } \\
\text { Al) }\end{array}$ \\
\hline $2-3$ & 26 & 0.399 & 0.440 & 0.460 & 0.488 \\
\hline $3-4$ & 27 & 0.420 & 0.464 & 0.471 & 0.501 \\
\hline $4-5$ & 28 & 0.440 & 0.486 & 0.482 & 0.511 \\
\hline $5-6$ & 29 & 0.459 & 0.508 & 0.491 & 0.521 \\
\hline $6-7$ & 30 & 0.479 & 0.530 & 0.499 & 0.530 \\
\hline $7-8$ & 31 & 0.498 & 0.552 & 0.508 & 0.538 \\
\hline
\end{tabular}

Rh). The beam spectra were computed following Ref. [34]. The tube voltage was tuned on the basis of the investigated compressed breast thickness. For instance, it was set to $26 \mathrm{kVp}$ for breast thickness ranging between $2 \mathrm{~cm}$ and $3 \mathrm{~cm}$ up to $31 \mathrm{kVp}$ for breast thickness in the range $7-8 \mathrm{~cm}$. Table 3 reports technique factors used for breast irradiations, including the 1st half value layer (HVL). The selected spectra and technique factors reflect those adopted in some commercial mammographic units $[36,37]$.

\subsection{MC code validation}

The Monte Carlo code, adopted in the case of homogeneous breast models, was largely validated versus both literature data and measurements $[19,21,23]$. In particular, it was validated versus data presented in the AAPM Task Group 195, cases I-III [35]. In Ref. [19], we showed that the differences between the results obtained with our code and those reported in the task group datasheet, for case 3 of the TG195, which simulated the MGD to the breast during a mammographic examination, are within the statistical uncertainty of the results.

As shown in previous papers [19,22], adopting heterogeneous breast phantoms with voxels randomly placed within the breasts and filled with either glandular tissue or adipose tissue, leads to different results, with respect to homogeneous phantoms composed of a homogeneous mixture of adipose and glandular tissue. In this paper, we compare MGD and glandular dose calculated by adopting standard homogeneous breast models to that evaluated with patient specific breast phantoms. Since the MC code here adopted for the glandular dose computation for heterogeneous breast phantoms is different from those of previous works $[19,22]$, we performed a further validation test. We produced 9 voxelised breast phantoms with a semi-circular cross section with a radius of $10 \mathrm{~cm}$ and with a skin layer of $0.2 \mathrm{~cm}$. The $0.5 \times 0.5 \times 0.5 \mathrm{~mm}^{3}$ voxels were made of a homogeneous mixture of adipose and glandular tissue for the homogeneous version of the breast phantom (Fig. 4a) and made either of glandular tissue or adipose tissue in the heterogeneous version (Fig. 4b). In this last case, the glandular voxels were randomly placed within the adipose tissue (Fig. 4b). Then we compared the MGD evaluated in the two cases. In the heterogeneous case, the breast phantom was replicated 9 times with a different random distribution of the glandular voxels (at a fixed glandular fraction of $20 \%$ by mass) and the average MGD was computed from the set of 9 simulations. For the tests described in this section, monoenergetic $\mathrm{X}$-ray beams ranging between $8 \mathrm{keV}$ and $39 \mathrm{keV}$ were simulated.

\section{Results}

\subsection{MC code validation}

Fig. 5a shows the percent ratio between the MGD evaluated with heterogeneous breast model in Fig. $4 \mathrm{~b}$ and that estimated with the homogeneous breast model in Fig. 4a. The homogenous model - which implies the use of the G-factor in Eq. (2) and the glandular and adipose tissue absorption coefficients fitting curves - leads to a MGD slightly larger than for the heterogeneous model, in agreement with previous papers $[19,22]$. The influence of the G-factor [22] is evident in Fig. 5b, which shows the percent ratio between the total dose (glandular dose plus adipose dose) calculated with the two models. Indeed, in Fig. 5b the G-factor is not employed and the dose differences are negligible. For photon energies lower than $10 \mathrm{keV}$, the differences in MGD is higher than $4 \%$ (ratio $=0.96$ ); however, the spectra usually adopted in mammography present effective energy higher than $15 \mathrm{keV}$ and related MGD differences lower than to 2\% [22].

\subsection{Homogeneous models vs. patient specific phantoms}

Fig. 6 shows the ratio between the MGD estimated via the homogeneous breast models \#1 and \#2 to that estimated by adopting the 20 patient specific digital breast phantoms described in the Section 2.3, for W/Al spectra. Model \#1 adopts a cylindrical breast model with a semicircular section and a skin layer made of adipose tissue and $5 \mathrm{~mm}$ thick; this produces a little difference in MGD estimates when compared to the patient specific phantoms, on average. In addition, for about $50 \%$ of the 


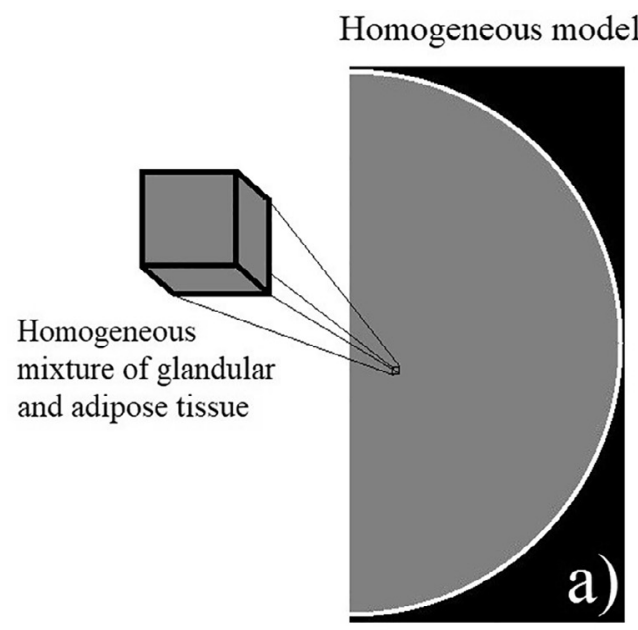

Heterogenous model

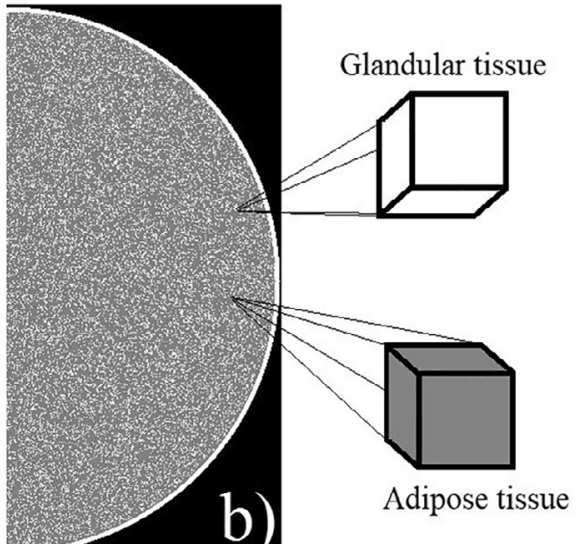

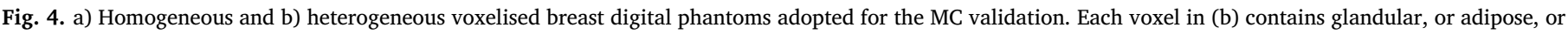
skin tissue.

considered cases (between 25th and 75th percentile) the MGD differs by $<5 \%$, in absolute terms, with respect to patient specific phantoms. However, the MGD underestimation with the homogeneous breast model \#1 is up to $33 \%$ at most; on the other hand, we found a maximum overestimation of $28 \%$ for a specific patient breast. A model with a skin layer of $4 \mathrm{~mm}$ made of skin tissue brings to lower MGD values [19]. This is the main cause of the reduction of the calculated MGD for the model \#2 (Fig. 6). Indeed, this model produces an average MGD underestimation of $11 \%$ when compared to patient specific breast phantoms with a maximum underestimation of $39 \%$ and a maximum overestimation of $14 \%$.

The results for the homogenous breast model with a $1.45 \mathrm{~mm}$ thick skin layer (models \#3 and \#4) do not differ largely from those obtained with the model \#1 (Fig. 7). In this case, the ratio between the MGD calculated with the homogeneous model \#3 and that calculated with the patient specific breast phantoms ranges between 0.69 and 1.30 (average value $=1.01$ ). The subcutaneous adipose layer shields the glandular tissue and brings to lower MGD values [19]: hence, the ratio between the MGD calculated by adopting model \#4 and that for the patient specific phantoms ranges between 0.66 and 1.26 (average value $=0.98)($ Fig. 7) .

Figs. 8 and 9 reproduce the results of Figs. 6 and 7 for W/Rh spectra. Fig. 8 shows the ratio between the MGD estimated via the homogeneous breast models \#1 and \#2 to that estimated by adopting the 20 patient specific digital breast phantoms. The difference between the model \#1 and patient specific breast phantoms remained unimportant, on average (Fig. 8): the first produced a mean MGD underestimation of $2 \%$. The minimum and maximum ratios between the MGD calculated for the model \#1 and that for the patient specific breast phantoms are 0.62 and 1.28 , correspondent to maximum underestimation and overestimation of $38 \%$ and $28 \%$, respectively. The average ratio between the MGD

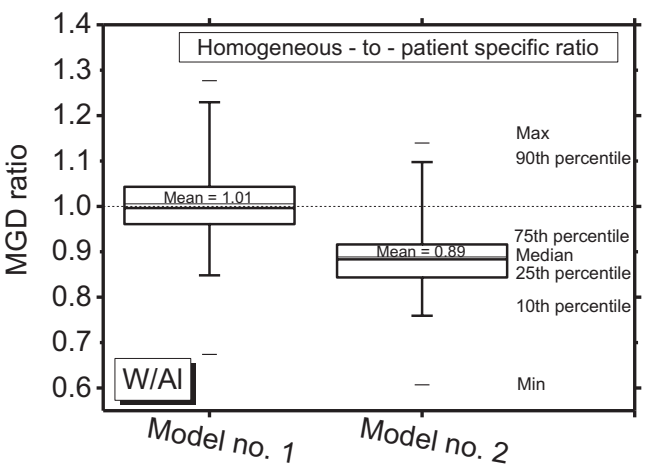

Fig. 6. Whiskers box plot of the ratio between the MGD estimated with the homogeneous models \#1 and \#2 to that estimated with the patient specific phantoms. W/Al spectra.

calculated for the model \#2 and for the patient specific phantoms also presents a slight reduction compared to the case in Fig. 6, and reveals an MGD of 14\% lower for the homogeneous case with a maximum underestimation of $43 \%$ and maximum overestimation of $13 \%$ within the investigated cohort. Larger differences were observed for the model \#3 in Fig. 9 when compared to data obtained for W/Al spectra. Indeed, while the model \#3 produced an average overestimation of $1 \%$ when compared to the structured phantoms for W/Al spectra, it produces an average underestimation of $8 \%$ when W/Rh spectra are adopted. In this last case, the ratio between the MGD evaluated with the homogeneous model and that for the patient specific phantom ranges between 0.59 and 1.21. For W/Rh spectra, the ratio between MGD calculated with the breast model \#4 and patient specific breasts is comprised in the range 0.64-1.31 with an average value of 0.99 (Fig. 9).
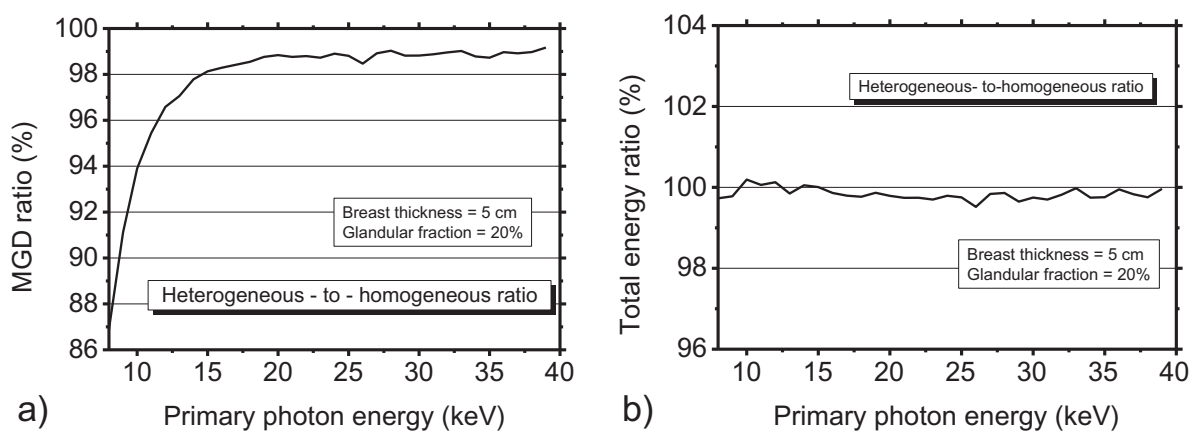

Fig. 5. a) Percent ratio between the MGD calculated with the heterogeneous breast model (Fig. 4b) and that evaluated for the homogeneous model (Fig. 4a). b) Percent ratio between the total dose (evaluated in both adipose and glandular tissue) calculated with the heterogeneous breast model (Fig. 4b) and that evaluated for the homogeneous model (Fig. 4a). 


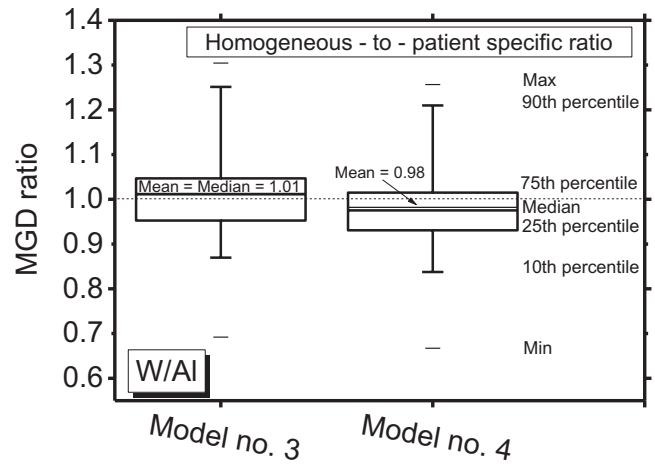

Fig. 7. Whiskers box plot of the ratio between the MGD estimated with the homogeneous models which present a 1.45 skin layer, either without (model \#3) or with (model \#4) the $2 \mathrm{~mm}$ subcutaneous adipose layer, to that estimated with the patient specific phantoms. W/Al spectra.

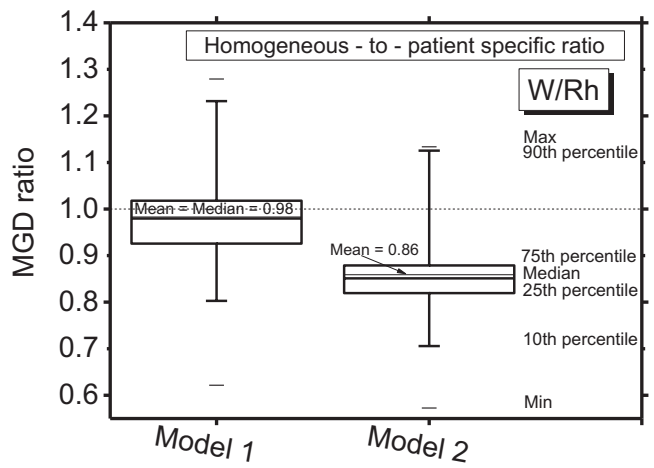

Fig. 8. Whiskers box plot of the ratio between the MGD estimated with the homogeneous models \#1 and \#2 to that estimated with the patient specific phantoms. W/Rh spectra.

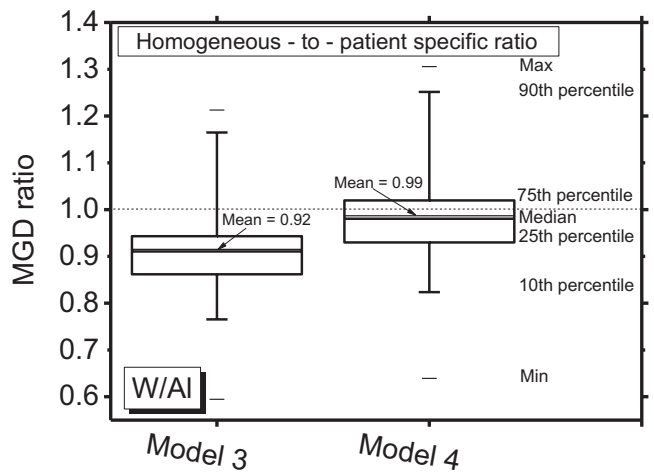

Fig. 9. Whiskers box plot of the ratio between the MGD estimated with the homogeneous models which present a 1.45 skin layer, either without (model \#3) or with (model \#4) the $2 \mathrm{~mm}$ subcutaneous adipose layer, to that estimated with the patient specific phantoms. W/Rh spectra.

\subsection{BreastSimulator's model vs. patient specific breast phantom}

Fig. 10 shows the glandular dose distribution within a patient specific breast phantom and that in a digital breast phantom produced with BreastSimulator. The two digital phantoms present the same glandular fraction by mass and the same compressed thickness. The volume histograms of glandular dose are reported in Fig. 11a. For this specific case, the MGD for the model produced via BreastSimulator is 33\% higher than that calculated for the patient specific phantom produced from 3D breast images. Indeed, in the first case the dose histogram mean value is $9.210^{-13} \mathrm{mGy}$ per photon (dose distribution standard deviation $=7.5$
$10^{-13} \mathrm{mGy}$ per photon; median $=7.210^{-13} \mathrm{mGy}$ per photon; $1 \mathrm{st}$ quartile $=1.610^{-13} \mathrm{mGy}$ per photon; 3rd quartile $=11.710^{-13} \mathrm{mGy}$ per photon) and in the latter it is $6.910^{-13} \mathrm{mGy}$ per photon (dose distribution standard deviation $=5.8 \quad 10^{-13} \mathrm{mGy}$ per photon; median $=3.910^{-13} \mathrm{mGy}$ per photon; 1 st quartile $=1.310^{-13} \mathrm{mGy}$ per photon; 3rd quartile $=8.110^{-13} \mathrm{mGy}$ per photon)

As shown in Fig. 10, the glandular tissue in the patient specific digital phantom produced from 3D breast images is mainly located in the inner part of the breast, and it is shielded from the radiation by the surrounding adipose tissue. On the other hand, the phantom produced via BreastSimulator presents a larger amount of glandular tissue close to the upper skin surface, for the same value of glandular fraction by mass. This glandular tissue receives a large dose, so contributing to the high dose tail in the histogram (Fig. 11a) and to a higher MGD value. In addition, the 3D dose spread resulted larger for the BreastSimulator phantom: indeed, the standard deviation of the glandular dose distribution was $29 \%$ higher, in this case (Fig. 11a). The dose spread evaluated as the ratio between the standard deviation and the mean of the glandular distribution - is $84 \%$ for the patient specific case and $82 \%$ for the BreastSimulator phantom. In the cumulative glandular dose distribution in Fig. 11b, the x-axis of the each histogram is normalized to the MGD value. This figure shows that, in both cases, about the $10 \%$ of the glandular tissue receives more than 2 times MGD and about the $44 \%$ of the total glandular tissue receives 0.5 times the MGD. It is worth noting that the MGD calculated with the breast model \#1 was $13 \%$ higher than that evaluated for the patient specific breast phantom adopted in this section.

\section{Discussion}

Protocols for breast dosimetry are based on simplified breast models $[6,8]$. There, the breast undergoing the mammographic examination is assumed to have a simple shape (cylinder with a semi-circular or semielliptical section) and made of a homogeneous mixture of adipose and glandular tissue enveloped in a layer mimicking the skin. These models do not consider neither the heterogeneous distribution of the gland (usually located at the inner part of the breast) nor the real breast silhouette. However, these features presented a huge influence on the MGD estimates [16,17]. The skin thickness - assumed of $5 \mathrm{~mm}$ in the actual EU, UK and USA protocols [2-4] and of $4 \mathrm{~mm}$ in the former USA protocols $[6,38]$ - is larger than $1.45 \mathrm{~mm}$ measured via high resolution $3 \mathrm{D}$ breast images $[18,26]$. Moreover, the breast silhouette, fixed and simple-shaped in the various protocols, can largely differ among breasts with similar thickness and glandular distribution. Such differences may lead to a bias in MGD estimates in the clinical practice and a benchmark is fundamental. For this reasons, we compared, via MC simulations, the MGD calculated by adopting simple breast models to that computed by means of patient specific digital breast phantoms. The investigated breast models were four (breast model \#1, \#2, \#3 and \#4) and presented different shapes and skin characteristics. The patient specific digital breast phantoms were produced from the scan of 20 high-resolution 3D uncompressed breasts acquired via a clinical breast dedicated CT scanner. The image voxels were classified as skin, adipose tissue or gland and the uncompressed breasts were digitally compressed via a mechanical model. The final compressed digital breast phantoms replicated the silhouettes and the heterogeneous glandular distributions of breasts undergoing mammography. These patient specific digital breast phantoms were assumed in this work as the reference for the MGD estimates. The breast model \#1 reflected the breast geometry proposed in Ref. [8] and adopted in the present UE, UK and USA protocols [2-4]. For W/Al spectra, it led to a negligible MGD overestimation (1\%), on average, when compared to patient specific digital breast phantoms; the maximum overestimation and underestimation calculated for specific breasts in the cohort were $28 \%$ and $33 \%$, respectively. On the other hand, the model \#2 - which had the same geometrical configuration of that adopted in Ref. [6] and in the former 


\section{Glandular dose, $10^{-13} \mathrm{mGy} /$ photon}

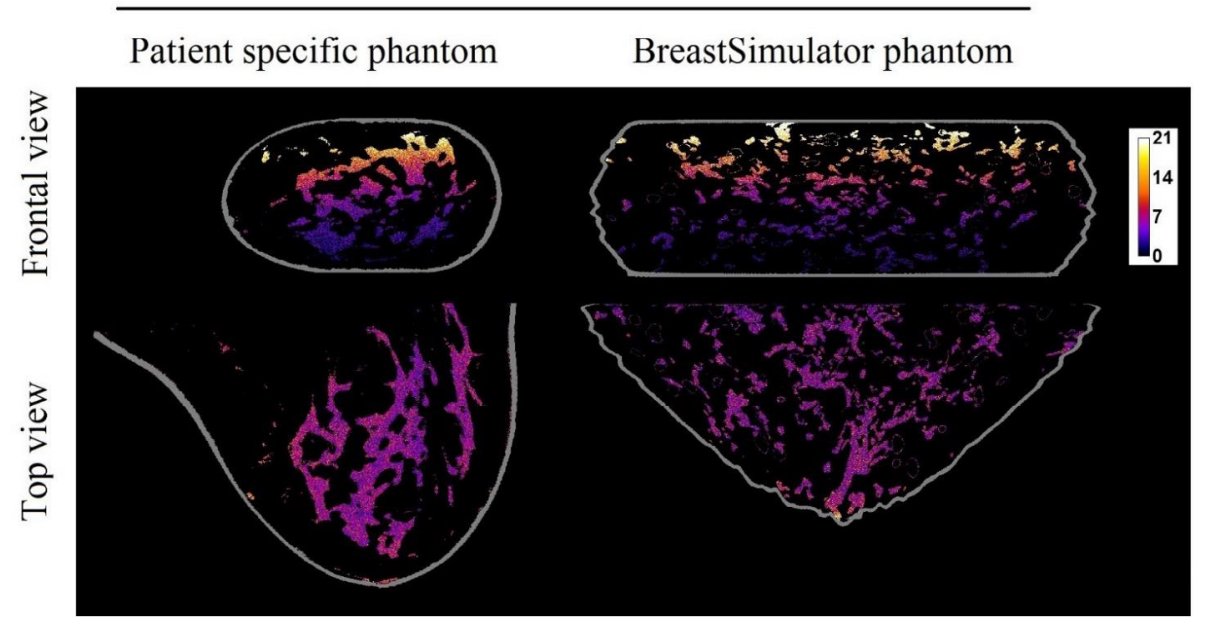

Fig. 10. Glandular dose distribution within the patient specific breast phantom (left) and within a digital breast phantom produced with BreastSimulator (right). W/Al spectrum at $30 \mathrm{kVp}$. Glandular fraction by mass $=16 \%$; breast thickness $=68.6 \mathrm{~mm}$. The light grey line represents the skin border.
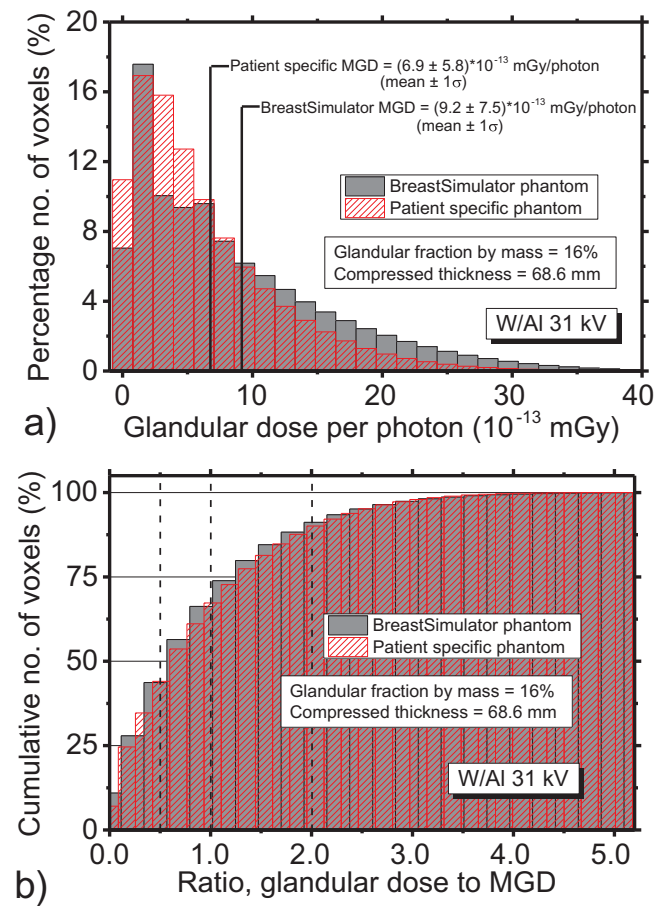

Fig. 11. a) Histograms of the glandular dose per photon in the voxels of the homogeneous and the patient specific breast phantoms. b) Cumulative dose distribution of the glandular dose in the two phantoms; the x-axis was normalized to the corresponding MGD of each phantom.

USA protocol for quality assurance in mammography [38] - led to lower MGD values. Indeed, it underestimated the MGD of patient specific phantoms, by $11 \%$, on average. In this case, the maximum overestimation and underestimation were $14 \%$ and $39 \%$, respectively. We investigated also a breast model with a semi-circular section and skin thickness of $1.45 \mathrm{~mm}$ (model \#3), which produced results similar to those obtained for the model \#1. Including an adipose layer between the 1.45-mm-thick skin layer and the glandular volume slightly reduced the MGD values, indeed for model \#4 the average MGD underestimation was $2 \%$, when compared to patient specific breast phantoms when $\mathrm{W} / \mathrm{Al}$ spectra were adopted. When $\mathrm{W} / \mathrm{Rh}$ spectra were adopted instead of W/Al anode/filter combination, no relevant differences were observed for the models \#1 and \#4, which underestimate the MGD of $2 \%$ and $1 \%$ respectively, when compared to patient specific breast phantoms. On the other hand, results for the model \#2 and \#3 showed increased underestimations with respect to the results for W/Al spectra. Indeed, from the average underestimations of $11 \%$ and $2 \%$ calculated for W/Al spectra, for W/Rh spectra the two homogeneous models produced underestimations of $14 \%$ and $8 \%$, respectively. In particular, the model \#3 maximum underestimation resulted of $41 \%$.

As alternative to standard homogeneous breast models, patient specific digital phantoms might be adopted for routine calculation, since they mimic the heterogeneous glandular distribution and breast shape. However, the lack of 3D digital breast phantoms produced from high-resolution in vivo images is the main limitation for the adoption of patient specific breast models in 2D breast dosimetry. On the other hand, digital breast phantoms replicating real breast anatomy and shape, can be created from the knowledge of the anatomical breast structures $[10,39]$. As alternative to the dosimetry which relies on the characteristics of the average breast by means of homogeneous breast models [1], a suitable digital breast phantoms, which reproduces the anatomical characteristics of the specific irradiated breast, such as the breast dimension and glandular mass fraction and localization, could be adopted for a patient specific dosimetry. In this work, we produced a digital breast phantom via the BreastSimulator software [20], which replicated the breast thickness and glandular fraction of one of the patient specific breast phantoms used for the models validation. From the comparison between the two digital phantoms (BreastSimulator vs. patient specific from BCT images) we showed that the former led to a mean value and a standard deviation of the glandular dose distribution higher than in the latter case, by $33 \%$ and $29 \%$, respectively. The highest glandular dose levels for the digital phantom produced via BrestSimulator were calculated for the glandular tissue close to the upper breast skin. In the corresponding patient specific breast phantom, the glandular tissue was located far from this region so resulting in a lower glandular dose levels, and hence lower MGD.

\section{Conclusions}

We compared the MGD evaluated, via MC simulations, for four homogeneous simple breast models vs. patient specific digital breast phantoms produced from high resolution 3D breast images, adopted as reference for two anode/filter combinations. A breast model which reflected that proposed in Ref. [8] did not lead to substantial differences in MGD estimates, on average. For W/Al spectra, the maximum 
calculated underestimation in the set of patient specific phantoms was $33 \%$, and the maximum calculated overestimation was $28 \%$. For the same anode/filter combination, a model with the characteristics of a homogeneous breast as proposed in Ref. [6], produced an average MGD underestimation of $11 \%$, with maximum underestimation and overestimation of $39 \%$ and $14 \%$, respectively. Adopting a homogeneous breast model with a skin thickness of $1.45 \mathrm{~mm}$ did not lead to substantial differences with respect to results for the model proposed in Ref. [8]. For W/Rh spectra, this last model led to MGD underestimation of $8 \%$, on average, when compared to patient specific breast phantoms and the maximum underestimation and overestimation over the entire patient specific breast cohort and over the four investigated homogeneous model resulted $43 \%$ and $31 \%$, respectively.

\section{Funding}

This work was partially supported by INFN (Istituto Nazionale di Fisica Nucleare), Italy, and by the MAXIMA project: "Three dimensional breast cancer models for X-ray imaging research". This project has received funding from the European Union's Horizon 2020 research and innovation programme under grant agreement No. 692097.

\section{References}

[1] Hammerstein RG, Miller DW, White DR, Masterson ME, Woodard HQ, Laughlin JS. Absorbed radiation dose in mammography. Radiology 1979;130:485-91.

[2] Institute of Physics and Engineering in Medicine (IPEM). The commissioning and routine testing of mammographic X-ray systems. IPEM Report 89. York, United Kingdom: IPEM; 2005.

[3] European Commission 2006 4th edition of the European Guidelines for Quality Assurance in Breast Cancer Screening and Diagnosis.

[4] ACR. Digital mammography quality control manual; 2016.

[5] Dance DR, Sechopoulos I. Dosimetry in X-ray-based breast imaging. Phys Med Biol 2016;61:R271-304.

[6] Wu X, Gary TB, Tucker DM. Spectral dependence of glandular tissue dose in screenfilm mammography. Radiology 1991;179:143-8.

[7] Boone JM. Glandular breast dose for monoenergetic and high-energy x-ray beams: Monte Carlo assessment. Radiology 1999;213:23-37.

[8] Dance DR. Monte-Carlo calculation of conversion factors for the estimation of mean glandular breast dose. Phys Med Biol 1990;35:1211-9.

[9] Dance DR, Skinner CL, Young KC, Beckett JR, Kotre CJ. Additional factors for the estimation of mean glandular breast dose using the UK mammography dosimetry protocol. Phys Med Biol 2000;45:3225-40.

[10] Dance DR, Hunt RA, Bakic PR, Maidment ADA, Sandborg M, Ullman G, et al. Breast dosimetry using high-resolution voxel phantoms. Rad Prot Dos 2005;114:359-63.

[11] Sarno A, Mettivier G, Russo P. Dedicated breast computed tomography: basic aspects. Med Phys 2015;42:2786-804.

[12] Mettivier G, Russo P. Measurement of the MTF of a cone-beam breast computed tomography laboratory scanner. IEEE Trans Nucl Sci 2011;58:703-13.

[13] Boone JM, Nelson TR, Lindfors KK, Seibert JA. Dedicated breast CT: radiation dose and image quality evaluation. Radiology 2001;221:657-67.

[14] Sarno A, Mettivier G, Golosio B, Oliva O, Spandre G, Di Lillo F, et al. Imaging performance of phase-contrast breast computed tomography synchrotron radiation and a CdTe photon-counting detector. Phys Med 2016;32:681-90.

[15] Boone JM, Hernandez AM, Seibert JA. Two-dimensional breast dosimetry improved using three-dimensional breast image data. Radiol Phys Technol 2017:10:129-41.

[16] Sechopoulos I, Bliznakova K, Qin X, Fei B, Feng SSJ. Characterization of the homogeneous tissue mixture approximation in breast imaging dosimetry. Med Phys 2012;39:5050-9.

[17] Hernandez AM, Seibert JA, Boone JM. Breast dose in mammography is about $30 \%$ lower when realistic heterogeneous glandular distributions are considered. Med Phys 2015;42:6337-48.

[18] Huang S-Y, Boone JM, Yang K, Kwan ALC, Packard NJ. The effect of skin thickness determined using breast CT on mammographic dosimetry. Med Phys 2008;35:1199-206.

[19] Sarno A, Mettivier G, Di Lillo F, Russo P. A Monte Carlo study of monoenergetic and polyenergetic normalized glandular dose $(\mathrm{DgN})$ coefficients in mammography. Phys Med Biol 2016;62:306-25.

[20] Bliznakova K, Sechopulos I, Buliev I, Pallikarakis N. BreastSimulator: a software platform for breast X-ray imaging research. J Biomed Graph Comput 2012;2:1-14.

[21] Sarno A, Mettivier G, Di Lillo F, Russo P. Monte Carlo evaluation of normalized glandular dose coefficients in mammography. International Workshop on Digital Mammography. Springer International Publishing: 2016. p. 190-6.

[22] Sarno A, Dance DR, van Engen RE, Young KC, Russo P, Di Lillo F, et al. A Monte Carlo model for mean glandular dose evaluation in spot compression mammography. Med Phys 2017;44:3848-60.

[23] Sarno A, Masi M, Antonelli N, Di Lillo F, Mettivier G, Castriconi R, et al. Dose volume distribution in digital breast tomosynthesis: a phantom study. Trans Rad Pl Med Sc 2017;1:322-8.

[24] Sarno A, Mettivier G, Russo P. Air kerma calculation in Monte Carlo simulations for deriving normalized glandular dose coefficients in mammography. Phys Med Biol 2017;62:N337-49.

[25] Kopans BD. Breast imaging. 3rd ed. Lippincott Williams \& Wilkins; 2007. p. 26-7.

[26] Shi L, Vedantham S, Karellas A, O'Connell AM. Technical Note: Skin thickness measurements using high-resolution flat-panel cone-beam dedicated breast CT. Med Phys 2013;40. 031913-1-6.

[27] Mettivier G, Bliznakova K, Sechopoulos I, Boone JM, Di Lillo F, Sarno A, et al. Evaluation of the BreastSimulator software platform for breast tomography. Phys Med Biol 2017;62:6446-66.

[28] Zyganitidis C, Bliznakova K, Pallikarakis N. A novel simulation algorithm for soft tissue compression. Med Biol Eng Comp 2007;45:661-9.

[29] Bliznakova K, Suryanarayanan S, Karellas A, Pallikarakis N. Evaluation of an improved algorithm for producing realistic 3D breast software phantoms: application for mammography. Med Phys 2010;37:5604-17.

[30] Kellner AL, Nelson TR, Cervino LI, Boone JM. Simulation of mechanical compression of breast tissue. IEEE Trans Biomed Eng 2007;54:1885-91.

[31] Yang X, Wu S, Sechopoulos I, Fei B. Cupping artifact correction and automated classification for high-resolution dedicated breast CT images. Med Phys 2012;39:6397-406.

[32] Fedon C, Longo F, Mettivier G, Longo R. GEANT4 for breast dosimetry: parameters optimization study. Phys Med Biol 2015;60:N311-23.

[33] Wilkinson L, Heggie CP. Glandular breast dose: potential errors. Radiology 2001:213.

[34] Boone JM, Fewell TR, Jennings RJ. Molybdenum, rhodium, and tungsten anode spectral models using interpolating polynomials with application to mammography. Med Phys 1997;24:1883-4.

[35] Sechopoulos I, Ali ES, Badal A, Badano A, Boone JM, Kyprianou IS, et al. Monte Carlo reference data sets for imaging research: executive summary of the report of AAPM Research Committee Task Group 195. Med Phys 2015;42:5679-91.

[36] Strudley CJ, Warren LM, Young KC. Technical evaluation of Siemens Mammomat Inspiration digital breast tomosynthesis system. NHSBSP Equipment Report 1306 ver.2; 2015.

[37] Marshall NW, Monnin P, Bosmans H, Bochud FO, Verdun FR. Image quality assessment in digital mammography: part I. Technical characterization of the systems. Phys Med Biol 2011;56:4201-20.

[38] Hendrick RE, Bassett L, Botsco MA, Deibel D, Feig S, Gray J. Mammography quality control manual. Reston, VA: American College of Radiology; 1999.

[39] Wang W, Qiu R, Ren L, Liu H, Wu Z, Li C, et al. Monte Carlo calculation of conversion coefficients for dose estimation in mammography based on a 3D detailed breast model. Med Phys 2017;44:2503-14. 